\title{
Supplemental information for Mediating Stochastic Switching of Single Molecules Using Chemical Functionality
}

Penelope A. Lewis, ${ }^{1}$ Christina E. Inman, ${ }^{2}$ Yuxing Yao, ${ }^{3}$ James M. Tour, ${ }^{3}$ James E. Hutchison, ${ }^{2}$ and Paul S. Weiss ${ }^{*, 1}$

Sample preparation. Commercial $\mathrm{Au}\{111\} /$ mica substrates were annealed with a hydrogen flame and then exposed to a $1 \mathrm{mM}$ ethanolic solution of 1ATC9 for $24 \mathrm{~h}$, rinsed thoroughly with ethanol, and dried under nitrogen. The fresh 1ATC9 monolayers were exposed to $\sim 1 \mathrm{~mL}$ of a $\sim 0.1 \mathrm{mM}$ THF solution of NPPB for 1 to $60 \mathrm{~s}$ in an inert atmosphere to perform the insertion. The NPPB/1ATC9 samples were imaged under ambient conditions using a beetle-style STM that has been detailed previously. (M. T. Cygan, M. T.; Dunbar, T. D.; Arnold, J. J.; Bumm, L. A.; Shedlock, N. F.; Burgin, T. P.; Jones, L.; Allara, D. L.; Tour, J. M.; Weiss, P. S. J. Am. Chem. Soc. 1998, 2721-2732).
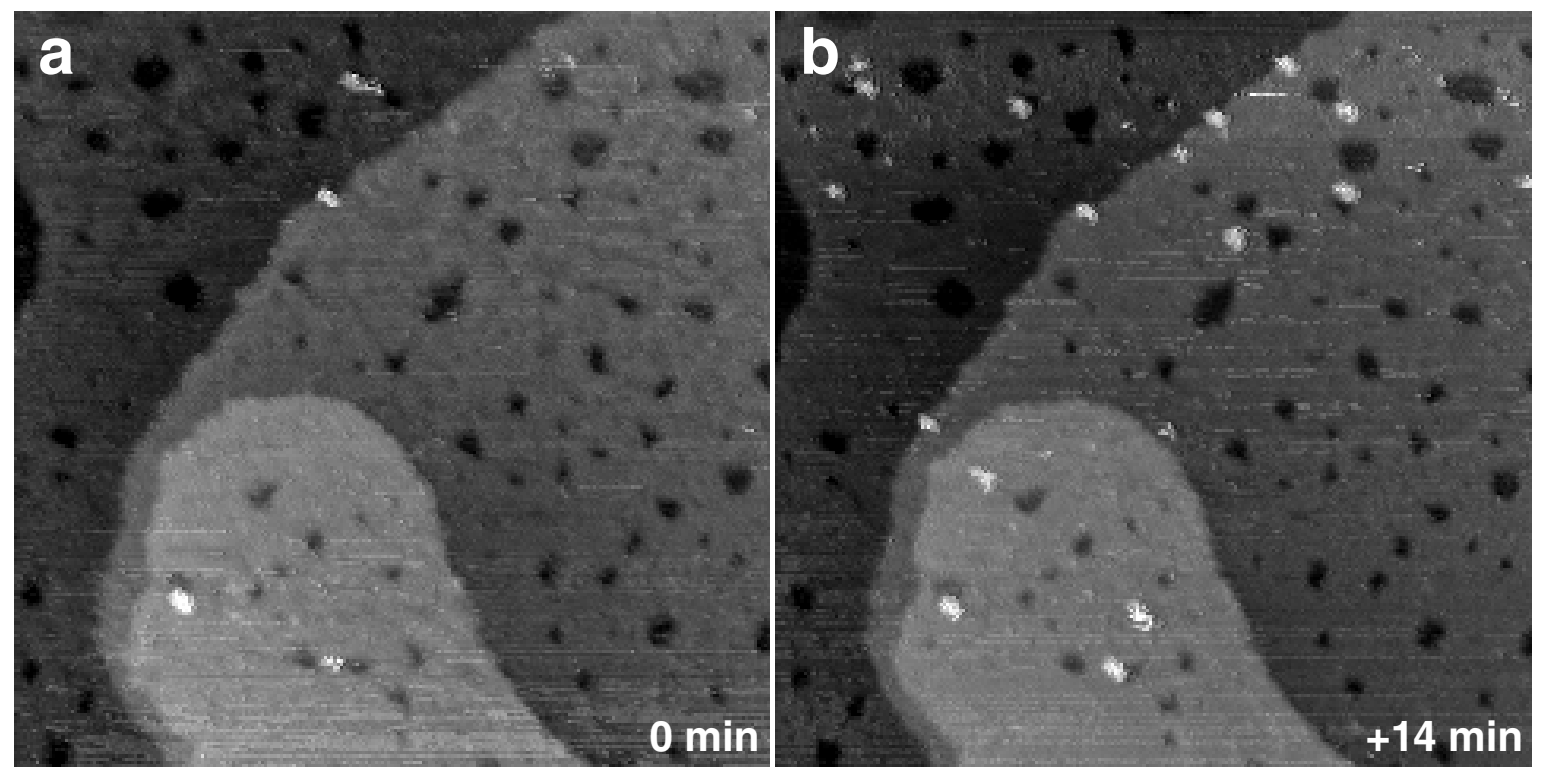

Supplemental Figure 1. (a) $1400 \AA \times 1400 \AA$ STM image taken at the end of a series of time-lapse images showing most of the NPPB molecules in the OFF state at negative sample bias. $\mathrm{V}_{\text {sample }}=-1 \mathrm{~V}, \mathrm{I}=2 \mathrm{pA}$. (b) $1400 \AA \times 1400 \AA \mathrm{STM}$ image taken in the same area 14 min after switching polarity to positive sample bias. $\mathrm{V}_{\text {sample }}=+1 \mathrm{~V}, \mathrm{I}=2$ pA. Some of the NPPB molecules have switched back to the ON state, demonstrating reversibility of bias-dependence switching. The reversibility shown here is not yet a precise method for controlling the state of a single NPPB molecule. Rather, it relies on the stochastic nature of the switching we observe in addition to exploiting the preference the NPPB molecules have for either the ON or OFF state depending on the applied electric field. As such, controlled switching between states exhibits some time dependence in addition to the applied bias dependence (i.e., the effect is not immediate once the bias polarity is reversed). 